\title{
Autosomal sexual dimorphism in methylation of advanced atherosclerotic carotid plaques
}

Gerard Pasterkamp $p^{1,2}$, Sander W. van der Laan ${ }^{2 *}$, Hester M. den Rujiter ${ }^{1 * \#}$.

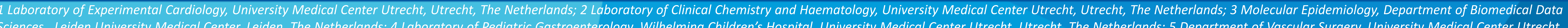
Sciences, Leiden University Medical Center, Leiden, The Netherlands; 4 Laboratory of Pediatric Gastroenterology, Wilhelmina Children's Hospital,

Background

Sex differences are evident in the diagnosis, prognosis and etiology of atherosclerosis. Women have more stable plaques in which plaque erosion is more often a substrate for cardiovascular disease, as compared to men where plaque rupture is dominant.

The extent to which these differences are correlated to DNA methylation (DNAm) in atherosclerotic plaques is not yet known.

Summary of results

After replication and meta-analysis (Figure 1), we identified 2,464 differentially methylated CpGs in plaques between the sexes, of which $82.0 \%$ are hypermethylated in men.

Genetic analyses showed that about $80 \%$ of these CpGs are also affected by genetic variation.

Smooth muscle cell content and intraplaque hemorrhage affect the majority of differentially methylated CpGs (Figure 2).

Sexual dimorphism in DNAm correlates with differential gene expression between sexes (Figure $3 \& 4)$.

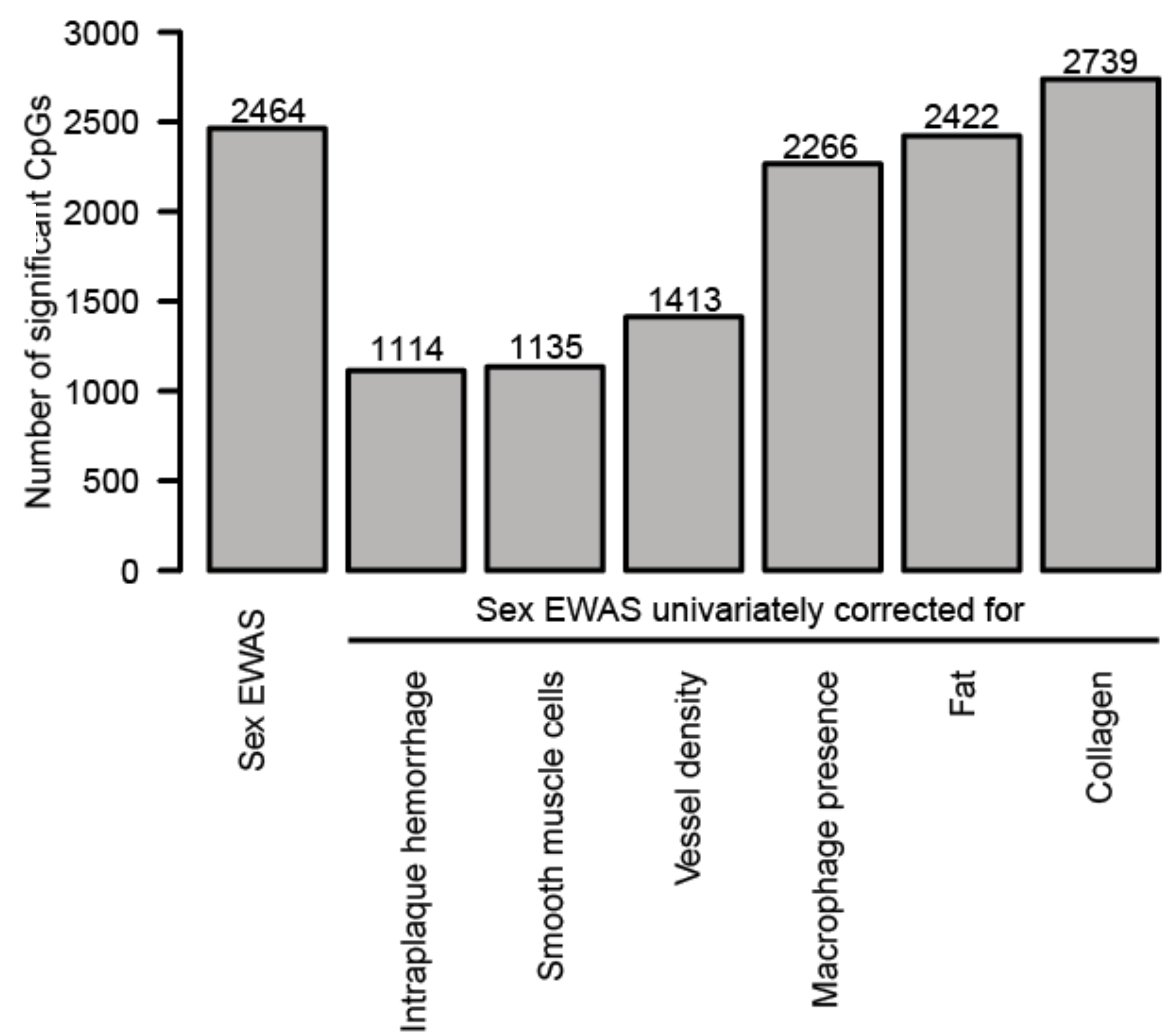

Figure 2 - Sexual dimorphism at hundreds of DNAm sites in carotid plaques.

Conclusions
Goal

To describe sexual dimorphic autosomal DNAm at CpG-dinucleotides (CpGs) in atherosclerotic plaques from carotid endarterectomy patients, we performed an epigenome-wide association study (EWAS) on sex.

To relate differences between sexes in DNAm to gene expression in single isolated cells from plaques.
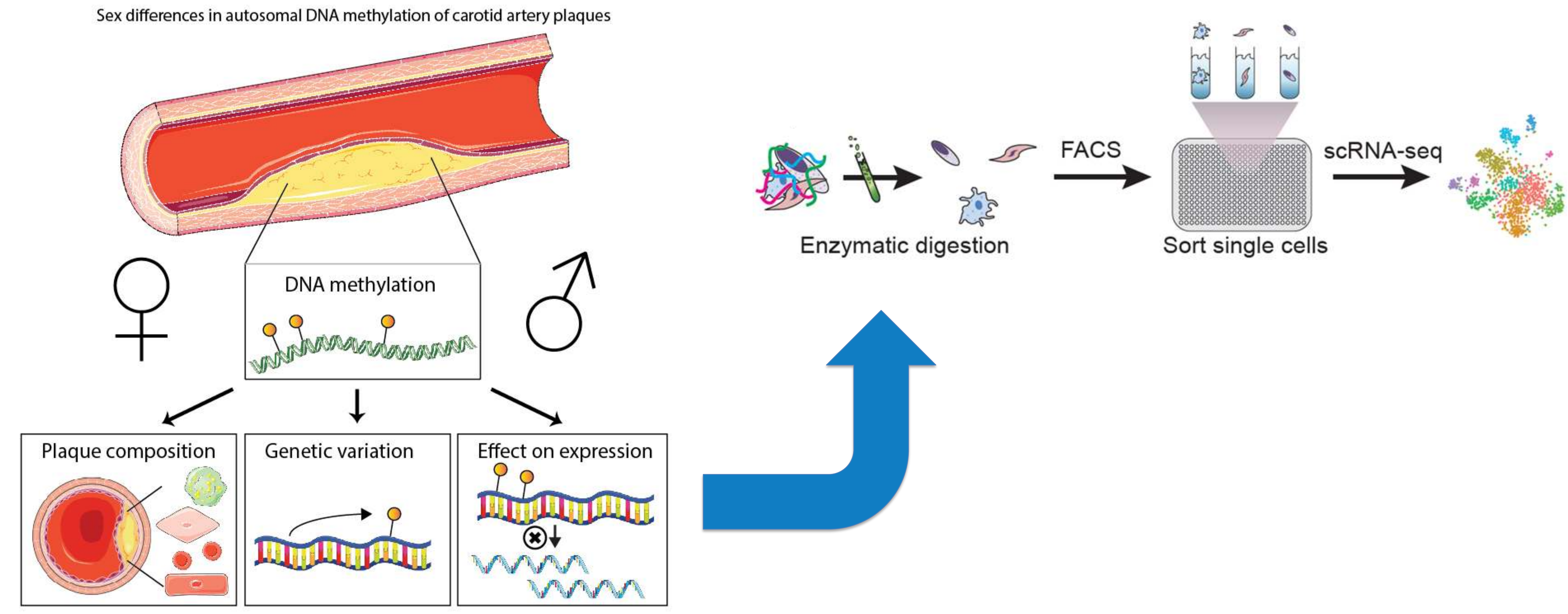

Figure 1 - General study design.

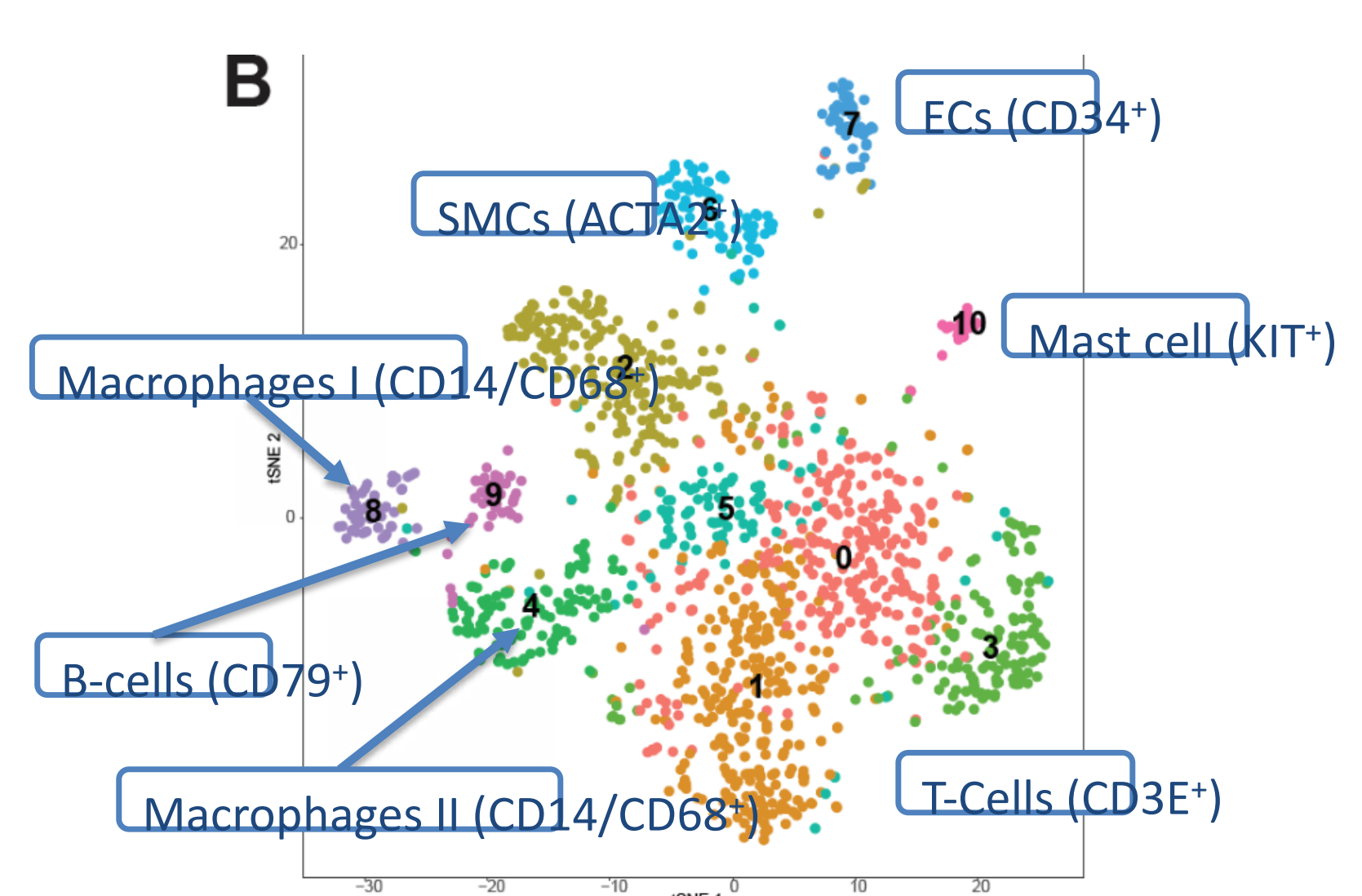

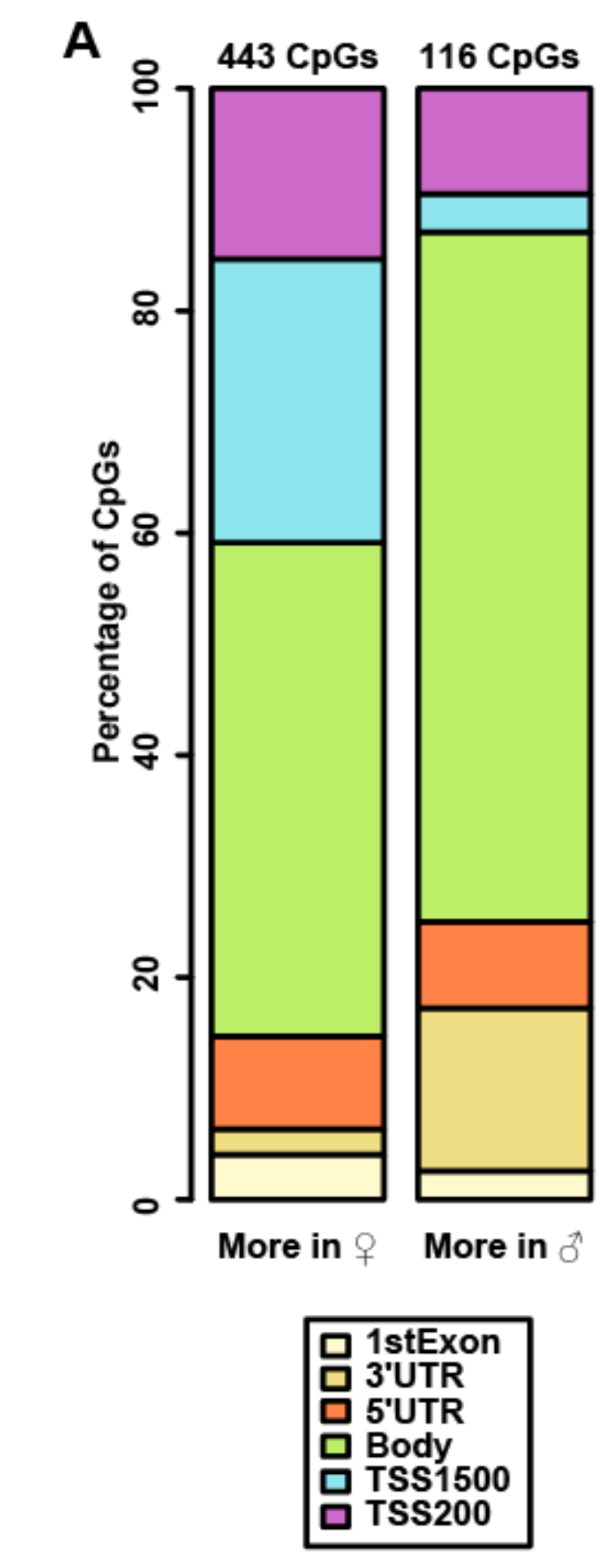

Figure 3 - DNAm at promotor sites differs between the sexes.
Methods

To describe sexual dimorphic autosomal DNAm at CpG dinucleotides (CpGs) in atherosclerotic plaques from carotid endarterectomy patients, we performed an epigenome-wide association study on $\operatorname{sex}(\% \mathrm{n}=180$, o $n=495$ ) corrected for age and hospital of inclusion. We applied a Bayesian method to control for latent bias and inflation (Bacon).

We associated genetic data $1000 \mathrm{G}$ phase 3 and GoNL5 imputed, with DNAm, using QTLToolKit, to assess the impact of genetic variation on sex differences. Carotid plaque composition was determined through histology and correlated to DNAm. Single cells were isolated from and correlated to DNAm. Sing
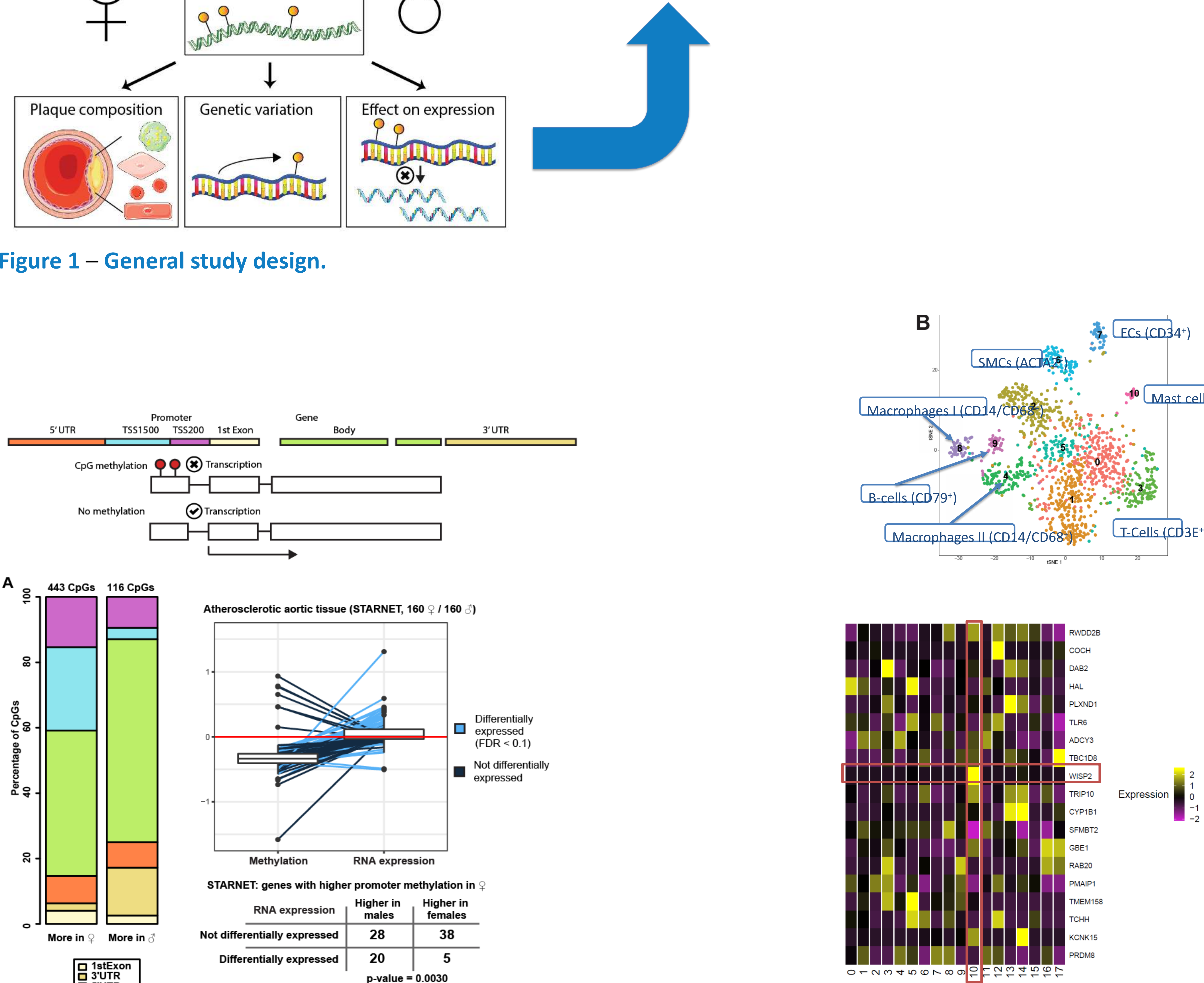

Figure 4 - Genes differentially methylated between the sexes at promotor sites, map to specific cellular communities in plaques.

DNAm in atherosclerotic plaques and points towards differences between sexes, partly explained by genetic variation and plaque composition.

DNAm of promotor regions in women correspond with lower gene expression in whole plaque and single cells derived from plaques.

These data combined may point to new biological cell-specific pathways that are different between sexes and relevant to atherosclerotic disease. 